\title{
Nitric Oxide Mediates Effects of Acute, not Chronic Naltrexone on LPS-Induced Hepatic Encephalopathy in Cirrhotic Rats
}

\begin{tabular}{|r|l|}
\hline Journal: & Canadian Journal of Physiology and Pharmacology \\
\hline Manuscript ID & cjpp-2016-0188.R1 \\
\hline Manuscript Type: & Article \\
\hline Complete List of Authors: & $\begin{array}{l}\text { Ghiassy, Bentolhoda ; Brain and Spinal Injury Research Center, Tehran } \\
\text { University of Medical Sciences, Tehran, Iran. } \\
\text { Rahimi, Nastaran; Experimental Medicine Research Center, Tehran } \\
\text { University of Medical Sciences, Department of Pharmacology, Tehran } \\
\text { University of Medical Scences, Tehran, Iran. } \\
\text { Javadi-Paydar, Mehrak; Tehran University of Medical Sciensec, Department } \\
\text { of Pharmacology } \\
\text { Gharedaghi, Mohammad Hadi; Tehran University of Medical Sciences, } \\
\text { Department of Pharmacology } \\
\text { Nourozi Javidan, Abbas; Tehran University of Medical Sciences, Tehran, } \\
\text { Iran., Brain and Spinal Cord Injury Research Center } \\
\text { Dehpour, Ahmad-Reza; Department of Pharmacology, School of Medicine, } \\
\text { Tehran University of Medical Sciences, Tehran, Iran, }\end{array}$ \\
\hline Keyword: & \begin{tabular}{l} 
Hepatic Encephalopathy, Opioids, Nitric Oxide, Cirrhosis, rat \\
\hline
\end{tabular} \\
\hline \multicolumn{2}{|c}{} \\
\hline
\end{tabular}

\section{SCHOLARONE"}

Manuscripts 


\section{Nitric Oxide Mediates Effects of Acute, not Chronic Naltrexone on LPS- Induced Hepatic Encephalopathy in Cirrhotic Rats}

Bentolhoda Ghiassy ${ }^{1 \mathrm{a}, \mathrm{b}}$, Nastaran Rahimi ${ }^{1 \mathrm{a}, \mathrm{c}}$, Mehrak Javadi-Paydar ${ }^{\mathrm{c}}$,, Mohammad Hadi Gharedaghi $^{\mathrm{a}, \mathrm{c}}$, Abbas Norouzi-Javidan ${ }^{\mathrm{b}}$, Ahmad R. Dehpour ${ }^{\mathrm{a}, \mathrm{c}}$ *

${ }^{a}$ Experimental Medicine Research Center, Tehran University of Medical Sciences, Tehran, Iran.

${ }^{\mathrm{b}}$ Brain and Spinal Injury Research Center, Tehran University of Medical Sciences, Tehran, Iran.

${ }^{c}$ Department of Pharmacology, School of Medicine, Tehran University of Medical Sciences, Tehran, Iran.

${ }^{1}$ First authors: Bentolhoda Ghiassy and Nastaran Rahimi contributed equally to this paper.

h.ghiasy100@gmail.com, Nastaran.rahimee@gmail.com, mehrakpaydar53@gmail.com, mhgharedaghi@gmail.com, norouziaj@yahoo.com,dehpour@yahoo.com

* Correspondence to: Prof. Ahmad Reza Dehpour, PharmD/PhD,

Department of Pharmacology, School of Medicine, Tehran University of Medical Sciences, Tehran, Iran. P.O. Box 13145-784.

Tel: +9821 88973652; fax: + 9821 66402569; e-mail: dehpour@yahoo.com; dehpoura@sina.tums.ac.ir

${ }^{\circledR}$ Current address for Dr. Javadi-Paydar is Committee on the Neurobiology of Addictive Disorders, The Scripps Research Institute, 10550 North Torrey Pines Road, La Jolla, CA. 


\section{Abstract}

Recent studies suggest endogenous opioids and nitric oxide (NO) are involved in the pathophysiology of hepatic encephalopathy (HE). In this study, the interaction between the opioid receptors antagonist, and NO was investigated on lipopolysaccharide (LPS)-induced HE in cirrhotic rats. Male rats were divided in the sham- and bile duct ligation (BDL)-operated groups. Animals treated with saline; naltrexone (10 mg/kg, i.p.); L-NAME (3 mg/kg, i.p.); alone or in combination by naltrexone. To induce HE, LPS (1 mg/kg, i.p.) was injected one hour after the final drug treatment. Hepatic encephalopathy scoring, hepatic histology and plasma NO metabolites levels and mortality rate were recorded. Deteriorated level of consciousness and mortality after LPS administration significantly ameliorated following both acute- and chronic treatment with naltrexone in cirrhotic rats. While, acute- and chronic administration of L-NAME did not change hepatic encephalopathy scores in cirrhotic rats. The effects of acute- but not chronic treatment of naltrexone on hepatic encephalopathy parameters were reversed by LNAME. Plasma NOx concentrations elevated in BDL rats, which were decreased after acute- and chronic treatment by naltrexone or L-NAME, significantly. We suggest both acute, and chronic treatment with naltrexone improved LPS-induced HE. But, only acute treatment with naltrexone may affect through NO pathway.

Keywords: Hepatic Encephalopathy, Opioids, Naltrexone, Nitric Oxide, Cirrhosis, Acute, Chronic, Rats. 


\section{Introduction}

Hepatic encephalopathy (HE) is a neuropsychiatric complication in patients with chronic or acute liver disease, which leads to changes in personality, intellectual function, and neuromuscular coordination (Butterworth 2000; Rodrigo et al. 2004). Although the exact pathophysiology of hepatic encephalopathy is not clearly understood, it is believed that this condition occurs due to alterations in the neurotransmitters and neuromodulators of the central nervous system, including endogenous opioids, nitric oxide, glutamate, gamma amino butyric acid and serotonin (Albrecht and Jones 1999; Butterworth 2001; Butterworth et al. 2009; Chu 2001; Yurdaydin et al. 1995; Yurdaydin et al. 1998). Moreover, in chronic liver disease there is an imbalance between inhibitory and excitatory neurotransmission with a shift towards inhibition. In this condition downregulation occurs in glutamate binding sites on the postsynaptic neurons and astrocytes which results in decreased cerebral glutaminergic tone (Michalak et al. 1996).

Opioids are neuroactive substances which are found in the central nervous system as well as in peripheral tissues. There is abundant evidence that plasma concentrations of endogenous opioids increase after liver failure (Albrecht and Jones 1999; Butterworth et al. 2009; Chu 2001). Increased opioid peptides contribute to different manifestations of chronic liver disease such as pruritus, ascitis, and hepatic encephalopathy, and it is evident that treatment with naltrexone reduces liver damage in animal models of cirrhosis (Yurdaydin et al. 1998; Yurdaydin rt al. 1996). Administration of opioid receptors antagonists such as naloxone and naltrexone ameliorates hepatic encephalopathy in rats with acute liver failure (Butterworth et al. 2009).

The metabolism of nitric oxide (NO) is altered in liver cirrhosis; and it has been suggested that these changes play a major role in the pathogenesis of HE (Clària et al. 1992; Hajrasouliha et al. 2005; Parekh and Balart 2015; Rao et al. 2002). The changes in the nitrogen production lead to multi-organ increase in the ammonia concentration, especially in plasma and central nervous 
system (CNS) leading to ammonia neurotoxicity (Parekh and Balart 2015; Shawcross et al. 2005). In rat models of chronic liver failure, it has been shown that ammonia impairs the glutamate-NO-cGMP pathway (Erceg et al. 2005). In addition, previous studies show chronic LNAME administration exerted protective effects on the severity of encephalopathy; in the process of fulminant hepatic failure development (Payabvash et al. 2007; Suarez et al. 2009). Interaction between opioids and NO has been shown in different biological models, such as morphine-induced analgesia, vascular hyporesponsiveness, and gastric damage in cholestatic rats (Babay et al. 1994; Nahavandi et al. 2001; Namiranian et al. 2001).

In addition, a subclinical hepatic encephalopathy is often unidentified in patients with cirrhosis, which negatively affects the activity of daily living e.g., driving a car or performing at work. The prevalence of subclinical hepatic encephalopathy is estimated to vary from $30 \%$ to 84\% (Citro et al. 2007; Groeneweg et al. 1998). Detecting effective factors on hepatic encephalopathy in patients with cirrhosis may help improve their quality of life. Interestingly, Wang et al reported that naltrexone decreased the overproduction of NO on acute hepatitis induced by LPS/D-galactoseamine in mice (Wang et al. 2008). Based on these facts, we aimed to evaluate the possible role of NO in the beneficial action of naltrexone on hepatic encephalopathy in cholestatic rats. 


\section{Methods}

\subsection{Housing and handling of the animals}

Male Albino-Wistar rats (Pasteur institute, Tehran, Iran), weighing 250 - 300 grams, were used in this study. All experiments and manipulations were carried out in accordance with the Guide for the Care and Use of Laboratory Animals (1996, published by National Academy Press, 2101 Constitution Ave. NW, Washington, DC 20055, USA) and our use of animals was reviewed and approved by the appropriate animal care review committee at the Department of Pharmacology, Tehran University of Medical Sciences. Rats were housed in an environment with a temperature of $22-23^{\circ} \mathrm{C}$, and a 12 hour light/dark cycle was provided. Animals had free access to tap water and a standard pellet chow. Each rat was used only once and each treatment group comprised 8 animals.

\subsection{Chemicals}

The following drugs were used in this study: naltrexone (opioid receptors antagonist), $\mathrm{N}^{\omega}$-nitroL-arginine methyl ester (L-NAME, nonspecific NO synthase [NOS] inhibitor), LPS (Escherichia Coli, serotype O127:B8), ketamine hydrochloride and xylazine hydrochloride. All of the drugs were purchased from Sigma (St. Louis, MO, USA), except for ketamine, xylazine and Griess reagent which were purchased from Gedoon Richter (Budapest, Hungary), Daroupakhsh (Tehran, Iran) and Alexis (Lausen, Switzerland), respectively. The drugs were dissolved in physiological saline and administered intraperitoneally (i.p.). Control groups received physiologic saline with a volume of $1 \mathrm{ml} / \mathrm{kg}$.

\subsection{Bile duct ligation surgical procedure}


Animals were randomly divided into sham-operated and bile duct ligated (BDL) groups. Bile duct ligations and sham operations were performed as previously described (Payabvash et al. 2007). Briefly, rats were anesthetized by i.p. injections of ketamine hydrochloride (100 mg/kg) and xylazine hydrochloride $(10 \mathrm{mg} / \mathrm{kg})$. After midline laparotomy, the bile duct was identified and ligated at two different locations with absorbable surgical threads. Thereafter, the bile duct was sectioned between the ligatures, and the abdominal wall was closed by sutures. All animals received $5 \mathrm{ml}$ of sterile physiologic saline i.p. after completion of surgery. Sham operation consisted of laparotomy, bile duct identification and manipulation without ligation.

\subsection{Experimental design}

Animals were randomly divided into 12 groups, each consisting of 8 rats. Groups 1 and 2 included sham-operated and BDL rats received saline as control groups.

Acute treatment groups were received following drug regimen: Groups 3 and 4 included sham operated and BDL rats that received naltrexone $(10 \mathrm{mg} / \mathrm{kg}$, i.p. $)$ on the 28 th day after surgery; in groups 5 and 6, sham-operated and BDL rats received a single injection of L-NAME (3 mg/kg, i.p.) on the 28th day after BDL; and finally, groups 7 and 8 included sham-operated and BDL rats that received an injection of naltrexone $(10 \mathrm{mg} / \mathrm{kg}$, i.p. $)+\mathrm{L}-\mathrm{NAME}(3 \mathrm{mg} / \mathrm{kg}$, i.p. $)$ on the 28th day after surgery.

Animal in chronic groups were treated daily with a specific drug regimen as described below: groups 9 and 10 included sham operated and BDL rats that received a daily injection of naltrexone $(10 \mathrm{mg} / \mathrm{kg}$, i.p.) for 28 days. To determine the involvement of the NO pathway in hepatic encephalopathy, in groups 11 and 12, sham-operated and BDL rats received a daily injection of L-NAME (3 mg/kg, i.p.) for 28 days; groups 13 and 14 included sham-operated and 
BDL rats that received a daily injection of naltrexone $(10 \mathrm{mg} / \mathrm{kg}$, i.p. $)+\mathrm{L}-\mathrm{NAME}(3 \mathrm{mg} / \mathrm{kg}$, i.p. $)$ for 28 days.

\subsection{Grading of hepatic encephalopathy and determination of mortality rates}

Encephalopathy was assessed every 20 minutes during the first 3 hours following LPS injection on the 28th day after surgery. Hepatic encephalopathy grades (I-IV) were assessed according hepatic encephalopathy scoring system uses basic reflexes (I(20-25), Lethargy; II(10-15), Lethargy and ataxia; III(15-20), Gradual loss of reflexes; IV(0-10), Coma) (Bruck et al. 1999). Additionally, mortality rates were determined 24 hours after LPS injection.

The effect of cirrhosis on hepatic encephalopathy was assessed by comparing hepatic encephalopathy scores between BDL and sham-operated rats after LPS injection on the 28th day after surgery.

\subsection{Histological studies of liver}

The liver was fixed with $4 \%$ paraformaldehyde and sectioned for evaluation based on microscopical (histologic) characters. Histological assessment of fibrosis, Ductular proliferation and inflammation were evaluated using hematoxylin and eosin (H\&E) as well as Masson trichrome staining. Fibrosis was staged $0-4$ based on Scheuer's scoring system ( 0 , no fibrosis; 1 , expansion of portal tract without linkage; 2, portal expansion with portal to portal linkage; 3 , extensive portal to portal and focal portal to central linkage; 4, cirrhosis) (Ebrahimkhani et al. 2006). Ductular proliferation and inflammation were graded on a scale of $0-4$, with $0,<10 \%$ of portal areas involved; $1,10-50 \%$ of portal areas involved; $2,>50 \%$ of portal areas involved; 3 , circumferential involvement of at least $50 \%$ of the portal area without significant expansion of portal tract; 4, circumferential involvement of at least $50 \%$ of the portal area with significant 
expansion of portal tract. The severity of changes was subjectively graded and compared with controls. Histological evaluation and scoring was carried out using a Zeiss ${ }^{\circledR}$ microscope equipped with an Olympus ${ }^{\circledR}$ colour video camera for digital imaging.

\subsection{Determination of plasma nitrite plus nitrate concentrations}

End products of NO (nitrite and nitrate) were measured through a colorimetric assay dependent upon the Griess reaction. One milliliter of blood was collected from tails of rats one hour after

drug injection on the $28^{\text {th }}$ day after surgery (immediately before LPS injection). Collection tubes contained ethylenediaminetetraacetic acid $(1.2 \mathrm{mg} / \mathrm{ml})$ to prevent blood from clotting. The collected blood was centrifuged for 10 minutes at $3400 \mathrm{~g}$, and plasma was extracted. One hundred and fifty microliters of plasma was pipetted into a 96 well microplate and diluted with 130 microliters of deionized water. Twenty microliters of Griess reagent was added to the diluted samples. Absorptions were read at a wavelength of $548 \mathrm{~nm}$ with a microplate ELISA reader (BioTek Instruments, Winooski, VT, USA). The concentration of nitrite plus nitrate $\left(\mathrm{NO}_{\mathrm{x}}\right)$ in different samples was determined by comparing the absorption of the samples with the absorption of sodium nitrite standard solutions (Rahimi et al. 2011).

\subsection{Statistical analysis}

All data (hepatic encephalopathy scores, mortality rates, plasma $\mathrm{NO}_{\mathrm{x}}$ levels) were analyzed with SPSS statistical software package (version 18.0, Chicago, IL, USA). Differences in hepatic encephalopathy scores were analyzed by repeated measures analysis of variance (ANOVA). Mortality rates were analyzed by chi-square analysis with Fisher's exact correction. Student's ttest and two-way ANOVA was employed to analyze differences in plasma $\mathrm{NO}_{\mathrm{x}}$ concentrations 
between groups. Differences with $P$ values less than 0.05 were considered as statistically significant.

\section{Results}

\subsection{Effects of cirrhosis on hepatic encephalopathy scores and mortality of rats}

Following LPS injection, the consciousness level of BDL rats markedly and progressively deteriorated, starting at 30 to 60 minutes after LPS injection. Three hours after LPS injection to BDL rats, high grade (grade 4) hepatic encephalopathy was observed. The observed changes in hepatic encephalopathy scores were statistically significant in comparison to sham-operated rats $(F(1,98)=169.36$, Fig. $1 ; P<0.001)$. Twenty four hours after LPS injection, the mortality rate of BDL rats was $100 \%$, which was also significantly higher than those of sham-operated rats $(\mathrm{P}$ $<0.01$ ). Sham-operated rats remained fully alert (Fig. 1) and did not die after LPS injection.

\subsection{Effects of acute treatment with naltrexone on hepatic encephalopathy grading and mortality of cirrhotic rats after LPS injection}

Acute treatment with naltrexone $(10 \mathrm{mg} / \mathrm{kg}$, ip) significantly decreased hepatic encephalopathy scores of BDL rats after LPS injection in comparison to saline-treated $\operatorname{BDL}$ rats $(F(1,89)=$ 114.01, Fig. 2; $P<0.001)$. Moreover, a single injection of naltrexone $(10 \mathrm{mg} / \mathrm{kg}$, ip) on the $28^{\text {th }}$ day after surgery reduced the mortality rate of BDL rats to $42.85 \%$ during the 24 hours after LPS injection, which was significantly lower than saline-treated BDL animals $(P<0.05)$.

\subsection{Effects of acute treatment with L-NAME and naltrexone on hepatic encephalopathy grading and mortality of cirrhotic rats after LPS injection}


Acute treatment with L-NAME (3 mg/kg, ip) had no effect on hepatic encephalopathy scores of BDL rats following LPS injection compared with saline treated BDL rats which received LPS $(F(1,89)=0.122$, Fig. $2 ; P>0.05)$. Additionally, all BDL animals which were treated with an acute dose of L-NAME ( $3 \mathrm{mg} / \mathrm{kg}$, ip) died within the first 24 hours following LPS injection, which was similar to saline-treated BDL rats.

Acute administration of L-NAME (3 mg/kg, ip) before naltrexone (10 mg/kg, ip) reversed the beneficial effects of acute treatment with naltrexone on hepatic encephalopathy scores of BDL rats $(F(1,98)=19.96$, Fig. $2 ; P<0.001)$. Furthermore, the mortality rate of BDL rats which received single doses of L-NAME and naltrexone was $87.5 \%$, which was significantly higher than the mortality rate of BDL rats that were injected with saline and naltrexone (mortality rate : $28.57 \% ; P<0.05)$.

\subsection{Effects of chronic treatment with naltrexone on hepatic encephalopathy scores and} mortality

Chronic treatment with naltrexone $(10 \mathrm{mg} / \mathrm{kg}$, ip) significantly reduced hepatic encephalopathy scores of cirrhotic rats after LPS injection in comparison to saline $(F(1,96)=76.28$, Fig. $3 ; P<$ 0.001). In addition, naltrexone reduced the mortality rate of BDL rats to $42.9 \%$ during the 24 hours following LPS injection compared with saline treated group $(P<0.05)$. Naltrexone had no effect when administered to sham-operated animals.

\subsection{Effects of chronic administration of L-NAME on hepatic encephalopathy scores and mortality of cirrhotic rats after LPS injection}

Chronic administration of L-NAME (3 mg/ $\mathrm{kg}$, ip) had no effect on consciousness of BDL and sham-operated rats after LPS injection $(F(1,98)=4.26$, Fig. $3 ; P>0.05)$. The mortality rate of 
L-NAME treated BDL animals was $85.71 \%$, which was not significantly different from that of saline-treated BDL animals.

Chronic co-administration of L-NAME (3 mg/kg, ip) and, naltrexone (10 mg/kg, ip) diminished hepatic encephalopathy scores of BDL rats, but had no effect on hepatic encephalopathy scores of naltrexone $(10 \mathrm{mg} / \mathrm{kg}$, ip) -treated BDL rats $(F(1,87)=0.039$, Fig. $3 ; P>0.05)$. Survival of BDL rats which were treated chronically with L-NAME and naltrexone was $28.57 \%$, which was also not significantly different from saline plus naltrexone-treated BDL rats.

\subsection{Histological analysis}

Liver sections showed significant morphological changes after 28 days of bile duct ligation, including extensive proliferation of the bile ducts, enlargement of the portal tracts and the formation of extensive periportal fibrosis as well as pericentral collagen deposition in comparison to sham-operated rats (Fig. $4 ; P<0.05$ ). As shown in tables 1 morphological change of all treated groups were significantly different by sham-operated groups. On the other hand, chronic administration of naltrexone and chronic co-administration of naltrexone and L-NAME reduced fibrosis and inflammation following bile duct ligation in comparison to BDL-operated rats, significantly $(P<0.05)$.

\subsection{Measurement of nitric oxide metabolites}

Table 2 provides information regarding plasma concentrations of NOx on day 28 after surgery in different experimental groups which received chronic drug treatments. The level of NOx was elevated in BDL rats in comparison with sham-operated controls $(F(1,11)=3.144, P<0.05)$. Administration of a single dose of L-NAME decreased the plasma NOx level in acute saline and 
naltrexone treated BDL rats, significantly $(P<0.05)$. In addition, chronic treatment with LNAME (3 mg/kg, i.p.), significantly abolished plasma concentrations rise of NOx in BDL rats $(P$ $<0.05)$. Also, chronic treatment with L-NAME reduced plasma NOx concentrations in chronic naltrexone treated group in BDL rats $(P<0.01)$.

\section{Discussion}

We found that acute and chronic administration of the opioid receptors antagonist, naltrexone, improves HE scores and reduces mortality rates following LPS injection in cirrhotic rats. Furthermore, both of acute and chronic administration of L-NAME, a nonselective NO synthase inhibitor, did not change the HE scores and mortality rates in BDL rats. Meanwhile improvement of hepatic encephalopathy scores and survival of cirrhotic rats by acute effect of naltrexone was reversed after treatment with L-NAME. On the other hand, administration of L-NAME did not inhibit the effect of chronic treatment with naltrexone on hepatic encephalopathy scores and mortality of cirrhotic rats, indicating that naltrexone exerts its acute effect on hepatic encephalopathy through a mechanism dependent on NO. These all were confirmed by liver histological experiments. In BDL rats, the plasma level of NO metabolites was higher compared with sham animals.

Hepatic encephalopathy is seen in patients with liver failure in function such as cirrhosis and spontaneous splenorenal shunt (Tarantino et al. 2009). Vascular Endothelial Growth Factordependent angiogenesis plays a crucial role in the formation of portal-systemic collateral vessels, which include Spleno-Renal shunts. Beyond the formation of portosystemic shunts, portal hypertention leads to Hepatic Encephalopathy. Transjugular Intrahepatic Portosystemic Shunt significantly impacts only on those patients with the extreme grade of ascites, although it 
worsens encephalopathy (Tarantino et al. 2009). Some patients with spontaneous shunts, who had undergone shunt reduction by a radiological approach, obtained an amelioration of HE (Kato et al. 2001). In addition, Elevated plasma levels of LPS contribute to induce HE in cirrhotic animals (Lindros et al. 2005) and this fact constitutes the basis for the use of LPS in animal models of HE (Harry et al. 1999; Wright et al. 2007). LPS induced a significant increase of hepatic and plasma levels of glutathione disulfide, an indicator of oxidant stress in mice (Galanos at al. 1979; Jaeschke et al. 1999). It has been shown that neutrophils-derived reactive oxygen species (ROS) are responsible for an intracellular oxidative stress in hepatocytes after LPS treatment. In addition, pro-inflammatory cytokines as TNF- $\alpha$ can induce the formation of ROS in hepatocytes (Adamson and Billings 1992).

Previous studies showed elevated levels of plasma endogenous opioids in cholestasis and cirrhosis contribute to different manifestations of chronic liver disease such as pruritus, ascitis, and hepatic encephalopathy (Bergasa and Jones 1992; Ebrahimkhani et al. 2008). Naltrexone as a long-acting opioid antagonist has been claimed to have anti-inflammatory and immunemodulatory effects both in vitro and in vivo (Greeneltch et al. 2004; Lin et al. 2005). In this study, both acute- and chronic administration of naltrexone lowered HE scores and mortality rates of cirrhotic animals. In line with our findings, Administration of naloxone or naltrexone in rats with thioacetamide-induced fulminate hepatic failure decreased the symptoms of HE (Yurdaydin et al. 1995). In addition, administration of naltrexone to BDL rats reduced liver fibrosis and hepatocyte apoptosis which is in consistent with previous studies (Payabvash et al. 2007; Ebrahimkhani et al. 2006). It has been shown that NO production increased in cirrhosis and in hepatotoxicity with bacterial endotoxemia (Finelli and Tarantino 2014), overproduction of NO can react with superoxide anions to form the peroxynitrite anion (ONOO-). In 1999, 
Cuzzocrea et al. proved that ONOO- can prompt damage in DNA, which can activate the polyadenylribosyl synthase and lead to ATP depletion causing multiple organ failure (Cuzzocrea et al. 1999). Peroxynitrite anion, also, decomposes to form hydroxyl radical which are responsible for cellular lipid peroxidation, protein oxidation, and mitochondrial impairment function, which cause further damage to tissues and can induce cell death (Kukreja and Hess 1992). Interestingly, naltrexone reduced the level of NO metabolites and superoxide anions in acute hepatitis induced by LPS/D-galactoseamine in mice (Wang et al. 2008). Then, the opioid receptors blockade is prominent and attributable in the survival after hepatic encephalopathy due to LPS.

The role of NO in the pathophysiology of HE is the controversial subject of ongoing debates. In this study, acute- and chronic administration of L-NAME per se, has no effect on HE scores and mortality rates of BDL rats. Consistent with previous report that chronic administration of LNAME did not alter HE scores in BDL rats (Chan et al. 2004). While, our results showed that increased NO metabolites levels, and hepatic fibrosis and inflammation in cirrhotic rats decreased in plasma and liver tissue of L-NAME treated animals. On the other hand, some authors believe that NO protected against hepatic encephalopathy, Chu et al determined that chronic L-NAME had detrimental effects on the severity of encephalopathy in TAA-treated rats (Chu et al. 2001; Chu et al. 2006), others suggest NO exacerbated hepatic encephalopathy. They suggested increased expression of NOS, may result from exposure to ammonia and/or manganese, which increase in brain due to chronic liver failure (Malecki et al. 1999; Rao et al. 2002; Rose et al. 1999; Suarez et al. 2009). In ammonia neurotoxicity, ammonia entering the brain is converted to glutamine in the astrocytes, a critical process for the onset of astrocyte damage (Albrecht and Norenberg 2006). Hyperammonemia has been shown to inhibit astrocytic 
GABA uptake, increase neuronal chloride currents by a direct action on the GABAA receptor complex, and potentiate the binding of GABAA and central benzodiazepine receptor agonists to the GABAA receptor complex (Albrecht and Jones 1999; Jones 2003; Schafer et al. 1999). In addition, exposure to ammonia causes an increase in brain lactate. In animal models presenting liver failure, a high lactate synthesis, which could be secondary to mitochondrial injury, has been shown (Bosoi et al. 2011). The excessive production of super Oxide (O2-) and nitrous Oxide (NO-) free radicals induces transient mitochondrial permeability pore (Rama et al. 2010) associated with astrocytic and neuronal damage, leading to energy impairment and cell death (Görg et al. 2010). Ammonia induced activation of mitogen-activated protein kinases and activation of nuclear factor- $\kappa \mathrm{b}$ (Norenberg et al. 2009).

Present study showed that a single injection of L-NAME reversed the protective effect of acutely administered naltrexone on HE. It has been shown that naltrexone reduced the overproduction of nitric oxide (NO) and superoxide anions induced by LPS/D-gal in mice (Wang et al. 2008). Acute administration of L-NAME reversed the beneficial effect of naltrexone in different biological models such as morphine-induced analgesia, vascular hyporesponsiveness, and gastric damage in cholestatic rats (Babay et al. 1994; Nahavandi et al. 2001; Namiranian et al. 2001; Rao et al. 2002). However in our study, chronic treatment with L-NAME did not alter the beneficial effect of 28-day treatment with naltrexone on HE scores. While, administration of LNAME significantly reduced NOx levels in cirrhotic rats, indicating that the alteration in NOx levels is not always in parallel with the manifestation of HE scoring. Also, chronic inhibition of NOS may operate additional signaling mechanisms that compensate for deficiency in NOS activity (Suarez et al. 2009). Interestingly, Ebrahimkhani et al reported that the increased nitrite/nitrate levels following cholestasis were not changed by chronic opioid receptor blockade. 
As well, they said the contribution of endogenous opioid system to vascular hyporesponsiveness in cirrhosis is not directly correlated to high plasma NO levels (Ebrahimkhani et al. 2008). Based on these data, we suggest that NO may affect only the beneficial effect of acute naltrexone administration on HE scores in rat.

In conclusion, this study showed that naltrexone alleviates LPS-induced hepatic encephalopathy and reduced mortality rate in BDL cirrhotic rats. Moreover, L-NAME reversed the beneficial effects of acute treatment with naltrexone on hepatic encephalopathy and mortality rate, suggesting that these effects are at least partially through augmentation of the plasma NO level; however, the amelioration observed on hepatic encephalopathy and mortality rate following the chronic treatment with naltrexone were not affected by L-NAME. We concluded that only acute, but not chronic, naltrexone administration may ameliorate LPS-induced hepatic encephalopathy through a NO pathway.

\section{Acknowledgement}

The study was supported by a grant from Experimental Medicine Research Center, Tehran University of Medical Sciences (grant no. 94-01-158-28590). 


\section{References:}

Adamson, G.M, and Billings, R.E. 1992. Tumor necrosis factor induced oxidative stress in isolated mouse hepatocytes. Arch. Biochem. Biophys. 294(1):223-229.

Albrecht, J, and Jones, E.A. 1999. Hepatic encephalopathy: molecular mechanisms underlying the clinical syndrome. J. Neurol. Sci. 170:138-146.

Albrecht, J, and Norenberg, M.D. 2006. Glutamine: a trojan horse in ammonia neurotoxicity. Hepatology, 44:788-794.

Babay, A.M., Kolesnikov, Y., Cheng, J., Inturrisi, C.E., Trifilletti, R.R, and Pasternak, G.W. 1994. Nitric oxide and opioid tolerance. Neuropharmacology, 33:1463-1470.

Bergasa, N.V, and Jones, E.A. 1992. Endogenous opioids accumulate in plasma in a rat model of acute cholestasis. Gastroenterology. 103:630-635.

Bosoi, C.R, and Rose, CF. 2014. Elevated cerebral lactate: Implications in the pathogenesis of hepatic encephalopathy. Metab. Brain Dis. 29(4):919-25.

Bruck, R., Aeed, H., Shirin, H., Matas, Z., Zaidel, L., Avni, Y, and Halpern, Z. 1999. The hydroxyl radical scavengers dimethylsulfoxide and dimethylthiourea protect rats against thioacetamide-induced fulminant hepatic failure. J Hepatol. 31:27-38.

Butterworth, R.F. 2000. Complications of cirrhosis III. Hepatic encephalopathy. J. Hepatol. 32: 171-180.

Butterworth, R.F. 2001. Neurotransmitter dysfunction in hepatic encephalopathy: new approaches and new findings. Metab Brain Dis. 16:55-65. 
Butterworth, R.F., Norenberg, M.D., Felipo, V., Ferenci, P., Albrecht, J, and Blei, A.T. 2009. Experimental models of hepatic encephalopathy: ISHEN guidelines. Liver Int. 29:783-188.

Chan, C.Y., Huang, S.W., Wang, T.F., Lu, R. H., Lee, F.Y., Chang, F.Y, and Lee, S.D. 2004. Lack of detrimental effects of nitric oxide inhibition in bile duct-ligated rats with hepatic encephalopathy. Eur. J. Clin. Invest. 34:122-128.

Chu, C.J., Chang, C.C., Wang, T.F., Lee, F.Y., Chang, F.Y., Chen, Y.C, and Lee, S.D. 2006. Detrimental effects of nitric oxide inhibition on hepatic encephalopathy in rats with thioacetamide-induced fulminant hepatic failure: Role of nitric oxide synthase isoforms. J Gastroenterol. Hepatol. 21(7):1194-1199.

Chu, C.J., Wang, S.S., Lee, F.Y., Chang, F.Y., Lin, H.C., Hou, M.C., et.al. 2001. Detrimental effects of nitric oxide inhibition on hepatic encephalopathy in rats with thioacetamide-induced fulminant hepatic failure. Eur. J. Clin. Invest. 31(2):156-63.

Citro, V., Milan, G., Tripodi, F.S., Gennari, A., Sorrentino, P., Gallotta, G., et.al. 2007. Mental status impairment in patients with West Haven grade zero hepatic encephalopathy: the role of HCV infection. J. of gastroenterol. 42:79-82.

Clària, J., Jiménez, W., Ros, J., Asbert, M., Castro. A., Arroyo, V., et al. 1992. Pathogenesis of arterial hypotension in cirrhotic rats with ascites: role of endogenous nitric oxide. Hepatology, 15: 343-349.

Cuzzocrea, S., Zingarelli, B., Costantino, G., Sottile, A., Teti, D, and Caputi, A.P. 1999. Protective effect of poly (ADP-ribose) synthetase inhibition on multiple organ failure after zymosan-induced peritonitis in the rat. Crit. Care Med. 27: 1517-1523. 
Ebrahimkhani, M.R., Moezi, L., Kiani, S., Merat, S, and Dehpour, A.R. 2008. Opioid receptor blockade improves mesenteric responsiveness in biliary cirrhosis. Dig. Dis. Sci. 53(11):30073011.

Ebrahimkhani, M.R., Kiani, S., Oakley, F., Kendall, T., Shariftabrizi, A., Tavangar, S.M, and Dehpour, A.R. 2006. Naltrexone, an opioid receptor antagonist, attenuates liver fibrosis in bile duct ligated rats. Gut. 55(11):1606-1616.

Erceg, S., Monfort, P., Hernandez-Viadel, M., Rodrigo, R., Montoliu, C, and Felipo V. 2005. Oral administration of sildenafil restores learning ability in rats with hyperammonemia and with portacaval shunts. Hepatology, 41(2): 299-306.

Finelli, C, and Tarantino, G. 2014. Non-alcoholic fatty liver disease, diet and gut microbiota. EXCLI j. 13: 461-490.

Galanos, C., Freudenberg, M.A, and Reutter, W. 1979. Galactosamine-induced sensitization to the lethal effects of endotoxin. Proceedings of the National Academy of Sciences. 76(11):59395943.

Görg, B., Qvartskhava, N., Bidmon, HJ., Palomero-Gallagher, N., Kircheis, G., Zilles, K, and Häussinger, D. 2010. Oxidative stress markers in the brain of patients with cirrhosis and hepatic encephalopathy. Hepatology, 52(1):256-265.

Greeneltch, K.M., Haudenschild, C.C., Keegan, A.D, and Shi, Y. 2004. The opioid antagonist naltrexone blocks acute endotoxic shock by inhibiting tumor necrosis factor- $\alpha$ production. Brain Behave. Immun. 18(5):476-484. 
Groeneweg, M., Quero, J.C., De Bruijn, I., Hartmann, I.J., Essink-bot, M.L., Hop, W.C, and Schalm, S.W. 1998. Subclinical hepatic encephalopathy impairs daily functioning. Hepatology, 28:45-49.

Hajrasouliha, A.R., Tavakoli, S., Jabehdar-Maralani, P., Ebrahimi, F., Shafaroodi, H., Mirkhani, S.H, and Dehpour, A.R. 2005. Cholestatic liver disease modulates susceptibility to ischemia/reperfusion- induced arrhythmia, but not necrosis and hemodynamic instability: the role of endogenous opioid peptides. J. Hepatol. 43:491-508.

Harry, D., Anand, R., Holt, S., Davies, S., Marley, R., Fernando, B, and Moore, K. 1999. Increased sensitivity to endotoxemia in the bile duct-ligated cirrhotic Rat. Hepatology, 301198 1205.

Jaeschke, H., Ho, Y.S., Fisher, M.A., Lawson, J.A. and Farhood, A. 1999. Glutathione peroxidase-deficient mice are more susceptible to neutrophil-mediated hepatic parenchymal cell injury during endotoxemia: importance of an intracellular oxidant stress. Hepatology, 29(2):443450.

Jones, EA. 2003. Potential mechanisms of enhanced GABA mediated inhibitory neurotransmission in liver failure. Neurochem. Int. 43(4-5): 509-516.

Kato, T., Uematsu, T., Nishigaki, Y., Sugihara, J., Tomita, E, and Moriwaki, H. 2001. Therapeutic effect of balloon-occluded retrograde transvenous obliteration on portal-systemic encephalopathy in patients with liver cirrhosis. Intern. med. 40:677-678. 
Kukreja, R.C, and Hess, M.L. 1992. The oxygen free radical system: from equations through membrane-protein interactions to cardiovascular injury and protection. Cardiovasc. Res. 26(7):641-655.

Lin, S.L., Lee, Y.M., Chang, H.Y., Cheng, Y.W. and Yen, M.H. 2005. Effects of naltrexone on lipopolysaccharide-induced sepsis in rats. J. Biom. Sci. 12(2):431-440.

Lindros, K.O, and Järveläinen, H.A. 2005. Chronic systemic endotoxin exposure: an animal model in experimental hepatic encephalopathy. Metab. Brain Dis. 20(4):393-8.

Malecki, E.A., Devenyi, A.G., Barron, T.F., Mosher, T.J., Eslinger, P., Flaherty-Craig, C.V, and Rossaro, L. 1999. Iron and manganese homeostasis in chronic liver disease: relationship to pallidal T1-weighted magnetic resonance signal hyperintensity. Neurotoxicology, 20(4):647-652.

Michalak, A., Rose, C., Butterworth, J, and Butterworth, RF. 1996. Neuroactive amino acids and glutamate (NMDA) receptors in frontal cortex of rats with experimental acute liver failure. Hepatology, 24(4): 908-913.

Nahavandi, A., Mani, A.R., Homayounfar, H., Akbari, M.R, and Dehpour, A.R. 2001. The role of the interaction between endogenous opioids and nitric oxide in the pathophysiology of ethanol-induced gastric damage in cholestatic rats. Fundam. Clin. Pharmacol. 15(3):181-187.

Namiranian, K., Samini, M., Mehr, S.E., Gaskari, S.A., Rastegar, H., Homayoun, H, and Dehpour, A.R. 2001. Mesenteric vascular bed responsiveness in bile duct-ligated rats: roles of opioid and nitric oxide systems. Eur. J. Pharmacol. 423(2):185-193.

Norenberg, MD., Rama, Rao KV, and Jayakumar, AR. 2009. Signaling factors in the mechanism of ammonia neurotoxicity. Metab. Brain Dis. 24(1): 103-117. 
Payabvash, S., Kiumehr, S., Nezami, B.G., Zandieh, A., Anvari, P., Tavangar, S.M, and Dehpour, A.R. 2007. Endogenous opioids modulate hepatocyte apoptosis in a rat model of chronic cholestasis: the role of oxidative stress. Liver Int. 27:538-547.

Parekh, P.J, and Balart, LA. 2015. Ammonia and its role in the pathogenesis of hepatic encephalopathy. Clin. Liver Dis. 19(3):529-37.

Rahimi, N., Dehpour, A.R., Javadi-Paydar, M., Sohanaki, H., Rabbani, S., Ansari, M, and Tafti, S.H.A. 2011. Effect of DETA-NONOate and papaverine on vasodilation of human internal mammary artery. Can. J. Physiol. Pharmacol. 89(12):945-951.

Rao, K.V.R., Reddy, P.V., Tong, X. and Norenberg, M.D. 2010. Brain edema in acute liver failure: inhibition by L-histidine. Am. J. Pathol. 176(3):1400-1408.

Rao, V.L. 2002. Nitric oxide in hepatic encephalopathy and hyperammonemia. Neurochem. Int. 41:161-170.

Rodrigo, R., Montoliu, C., Chatauret, N., Butterworth, R., Behrends, S., Del Olmo, J.A, et. al. 2004. Alterations in soluble guanylate cyclase content and modulation by nitric oxide in liver disease. Neurochem. Int. 45(6): 947-953.

Rose, C., Butterworth, R.F., Zayed, J., Normandin, L., Todd, K., Michalak, A, and PomierLayrargues, G. 1999. Manganese deposition in basal ganglia structures results from both portalsystemic shunting and liver dysfunction. Gastroenterology. 117(3):640-4.

Schafer, DF., Fowler, JM., Munson, PJ., Thakur, AK., Waggoner, JG, and Jones, EA. 1983. Gamma-aminobutyric acid and benzodiazepine receptors in an animal model of fulminant hepatic failure. J. Lab. Clin. Med. 102(6): 870-880. 
Shawcross, D, and Jalan, R. 2005. The pathophysiologic basis of hepatic encephalopathy: central role for ammonia and inflammation. Cell Mol. Life Sci. 62(19-20):2295-304.

Suarez, I., Bodega, G., Rubio, M, and Fernandez, B. 2009. Induction of NOS and nitrotyrosine expression in the rat striatum following experimental hepatic encephalopathy. Metab. Brain Dis. 24: 395-408.

Tarantino, G., Citro, V., Conca, P., Riccio, A., Tarantino, M., Capone, D, e. al. 2009. What are the implications of the spontaneous spleno-renal shunts in liver cirrhosis?.BMC Gastroenterol. 9(1):89-98.

Wang, C.C., Cheng, P.Y., Peng, Y.J., Wu, E.S., Wei, H.P. and Yen, M.H. 2008. Naltrexone protects against lipopolysaccharide/D-galactosamine-induced hepatitis in mice. J. Pharmacol. Sci. 108(3):239-247.

Wright, G., Davies, N.A., Shawcross, D.L., Hodges, S.J., Zwingmann, C., Brooks, H.F, and Jalan, R. 2007. Endotoxemia produces coma and brain swelling in bile duct ligated rats. Hepatology, 45:1517-1526.

Yurdaydin, C., Karavelioglu, D., Onaran, O., Celik, T., Yasa, M.H, and Uzunalimoglu, O. 1998. Opioid receptor ligands in human hepatic encephalopathy. J. Hepatol. 29:796-801.

Yurdaydin, C., Herneth, A.M., Puspok, A., Steindl, P., Singer, E, and Ferenci, P. 1996. Modulation of hepatic encephalopathy in rats with thioacetamide-induced acute liver failure by serotonin antagonists. Eur. J. Gastroenterol. Hepatol. 8:667-671.

Yurdaydin, C., Li, Y., Ha, J.H., Jones, E.A., Rothman, R, and Basile, A.S. 1995. Brain and plasma levels of opioid peptides are altered in rats with thioacetamide-induced fulminant hepatic failure: implications for the treatment of hepatic encephalopathy with opioid antagonists. J. Pharmacol. Exp. Ther. 273:185-192. 


\section{Table 1.}

a) Histological assessment of fibrosis in rats after 28 days surgery.

\begin{tabular}{|c|c|c|c|c|c|c|c|}
\hline Surgery & Drug regimen & Stage 0 & Stage 1 & Stage 2 & Stage 3 & Stage 4 & Mean (SD) \\
\hline Sham opeated & Saline & 8 & 0 & 0 & 0 & 0 & $0.00(0.00)$ \\
\hline \multirow[t]{4}{*}{ Bile duct ligated } & Saline (Chronic) & 0 & 1 & 4 & 2 & 1 & $2.37(0.31)^{* *}$ \\
\hline & Naltrexone (Acute) & 0 & 2 & 3 & 3 & 0 & $2.12(0.83)^{*}$ \\
\hline & L-NAME) (Acute) & 0 & 1 & 2 & 4 & 1 & $2.62(0.91)^{*}$ \\
\hline & L-NAME + Naltrexone (Acute) & 0 & 1 & 3 & 4 & 0 & $2.37(0.74)^{* *}$ \\
\hline \multicolumn{8}{|l|}{ Bile duct ligated } \\
\hline & Naltrexone (Chronic) & 5 & 0 & 2 & 1 & 0 & $0.87(0.12)^{*} 7$ \\
\hline & L-NAME) (Chronic) & 0 & 0 & 3 & 3 & 2 & $2.87(0.83)^{* *}$ \\
\hline & L-NAME + Naltrexone (Chronic) & 4 & 1 & 2 & 1 & 0 & $1.00(0.81)^{* \neq}$ \\
\hline
\end{tabular}


b) Histological assessment of inflamation in rats after 28 days surgery.

\begin{tabular}{|c|c|c|c|c|c|c|c|}
\hline Surgery & Drug regimen & Stage 0 & Stage 1 & Stage 2 & Stage 3 & Stage 4 & Mean (SD) \\
\hline Sham opeated & Saline & 8 & 0 & 0 & 0 & 0 & $0.00(0.00)$ \\
\hline \multirow[t]{4}{*}{ Bile duct ligated } & Saline (Chronic) & 0 & 1 & 4 & 2 & 1 & $1.75(0.08)^{* *}$ \\
\hline & Naltrexone (Acute) & 0 & 2 & 3 & 3 & 0 & $0.05(0.08)^{*}$ \\
\hline & L-NAME) (Acute) & 0 & 1 & 2 & 4 & 1 & $1.12(0.28)^{* *}$ \\
\hline & L-NAME + Naltrexone (Acute) & 0 & 1 & 3 & 4 & 0 & $1.12(0.43)^{* *}$ \\
\hline
\end{tabular}

Bile duct ligated

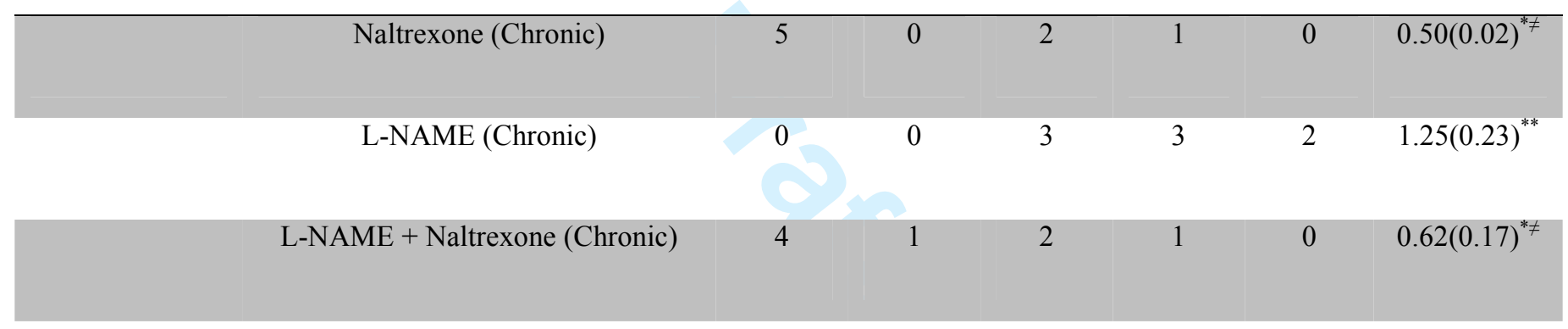

Each group composed of 8 rats. Data are represented as mean \pm S.D. * $\mathrm{P}<0.05$ in comparison to the sham-operated group. ${ }^{\#} \mathrm{P}<0.05$ in comparison to the saline-treated bile duct ligated group. 


\section{Table 2.}

Plasma concentrations of $\mathrm{NO}_{\mathrm{x}}$ in rats after 28 days surgery.

\begin{tabular}{|c|c|c|}
\hline Surgery & Drug regimen & $\begin{array}{c}\mathrm{NO}_{\mathrm{x}} \text { Concentration } \\
(\mu \mathrm{m}\end{array}$ \\
\hline Sham opeated & Saline & $18.86 \pm 1.53$ \\
\hline \multirow[t]{4}{*}{ Bile duct ligated } & Saline (Chronic) & $26.78 \pm 6.96^{*}$ \\
\hline & Naltrexone (Acute) & $32.56 \pm 9.78^{\neq}$ \\
\hline & L-NAME (Acute) & $16.55 \pm 4.73^{\neq}$ \\
\hline & L-NAME + Naltrexone (Acute) & $17.62 \pm 7.46^{\text {\&\& }}$ \\
\hline \multirow[t]{4}{*}{ Bile duct ligated } & Saline (Chronic) & $27.56 \pm 7.96^{*}$ \\
\hline & Naltrexone (Chronic) & $37.89 \pm 10.35^{\neq}$ \\
\hline & L-NAME (Chronic) & $16.32 \pm 6.57^{\neq}$ \\
\hline & L-NAME + Naltrexone (Chronic) & $21.36 \pm 7.24^{\& \&}$ \\
\hline
\end{tabular}

Each group composed of 8 rats. Data are represented as mean \pm S.E.M. $* \mathrm{P}<0.05$ in comparison to the sham-operated group. ${ }^{\#} \mathrm{P}<0.05$ and ${ }^{\# \#} \mathrm{P}<0.01$ in comparison to the saline-treated bile duct ligated group. ${ }^{\& \&} \mathrm{P}<0.01$ in comparison to the naltrexone- treated bile duct ligated group. 


\section{Figure Captions}

Fig. 1 Effect of LPS injection on hepatic encephalopathy scores in cirrhotic rats compared with sham- operated rats. These groups were treated with saline for 28 days after surgery. On day 28 , hepatic encephalopathy grading was assessed (based on Bruck study criteria) every 20 minutes during the first 3 hours following LPS injection.Data are presented as mean \pm S.E.M. Each group consisted of 8 rats and differences in hepatic encephalopathy scores were analyzed by repeated measures analysis of variance (ANOVA). ** $P<0.01$ and $* * * P<0.001$ in comparison to the corresponding control group.

Fig. 2 Effects of chronic administration of naltrexone, L-NAME and co-administration of naltrexone and L-NAME on LPS-induced hepatic encephalopathy in cirrhotic rats. Saline, Naltrexone (10 mg/kg, ip), and L-NAME (3 mg/kg, ip) were administered daily for 28 days after BDL. L-NAME (3 $\mathrm{mg} / \mathrm{kg}), 15$ minutes before naltrexone (10 $\mathrm{mg} / \mathrm{kg})$ were administered daily for 28 days after surgery. One hour after the last injections on day 28, LPS (1 $\mathrm{mg} / \mathrm{kg}$, ip) was injected then hepatic encephalopathy grading was assessed throughout a 3-hour session. Data are presented as mean \pm S.E.M. Each group consisted of 8 rats and differences in hepatic encephalopathy scores were analyzed by repeated measures analysis of variance (ANOVA). ${ }^{\times}$Significant differences between naltrexone- and saline-treated groups, 40-100 minutes after LPS injection $(P<0.01)$ and $100-180$ minutes after LPS injection $(P<$ 0.001). ${ }^{+}$Significant differences between (L-NAME + naltrexone)- and saline-treated groups 40100 minutes after LPS injection $(P<0.01)$ and 100-180 minutes after LPS injection $(P<0.001)$. 
Fig. 3 Effects of acute administration of naltrexone, L-NAME and co-administration of naltrexone and L-NAME on LPS-induced hepatic encephalopathy in cirrhotic rats. Saline, Naltrexone (10 mg/kg, ip), and L-NAME (3 mg/kg, ip) were administered day 28 days. L-NAME (3 mg/kg), 15 minutes before naltrexone $(10 \mathrm{mg} / \mathrm{kg})$ were administered on day 28 after surgery. One hour after the injections, LPS (1 $\mathrm{mg} / \mathrm{kg}$, ip) was injected then hepatic encephalopathy grading was assessed throughout a 3-hour session. Data are presented as mean \pm S.E.M. Each group consisted of 8 rats and differences in hepatic encephalopathy scores were analyzed by repeated measures analysis of variance (ANOVA). ${ }^{\times}$Significant differences were observed between naltrexone- and saline-treated groups 40-100 minutes after LPS injection $(P<0.01)$ and $100-180$ minutes after LPS injection $(P<0.001) .+$ Significant differences were observed between naltrexone- and saline-treated groups 100-180 minutes after LPS injection $(P$ $<0.001)$

Fig. 4 Haematoxylin and eosin stained sections of sham and bile duct ligated (BDL) liver tissues in rats. Twenty eight days after the surgical procedure, liver tissue was obtained and collagen fibers were stained with Masson's Trichrome. Bile duct ligation was associated with extensive bridging fibrosis (portal to portal and portal to central linkage with fibrotic bands) and bile duct proliferation. A. Sham-operated rats, B. BDL rats, C. chronic treatment with naltrexone of BDL rats, D. acute treatment with naltrexone of BDL rats, E. acute treatment with L-NAME + naltrexone of BDL rats, and F. chronic treatment with L-NAME + naltrexone of BDL rats. 


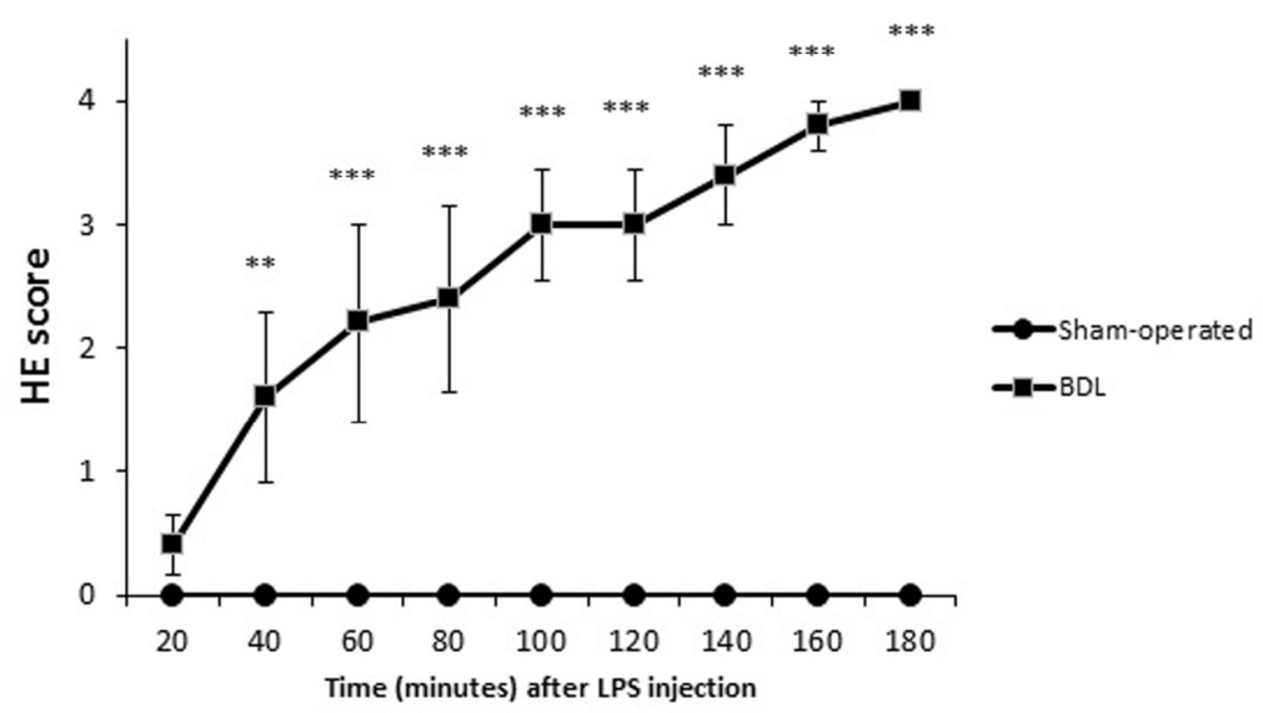

Fig. 1 Effect of LPS injection on hepatic encephalopathy scores in cirrhotic rats compared with shamoperated rats. These groups were treated with saline for 28 days after surgery. On day 28, hepatic encephalopathy grading was assessed (based on Bruck study criteria) every 20 minutes during the first 3 hours following LPS injection.Data are presented as mean \pm S.E.M. Each group consisted of 8 rats and differences in hepatic encephalopathy scores were analyzed by repeated measures analysis of variance (ANOVA). $* * \mathrm{P}<0.01$ and $* * * \mathrm{P}<0.001$ in comparison to the corresponding control group.

$$
83 \times 54 \mathrm{~mm}(300 \times 300 \text { DPI })
$$




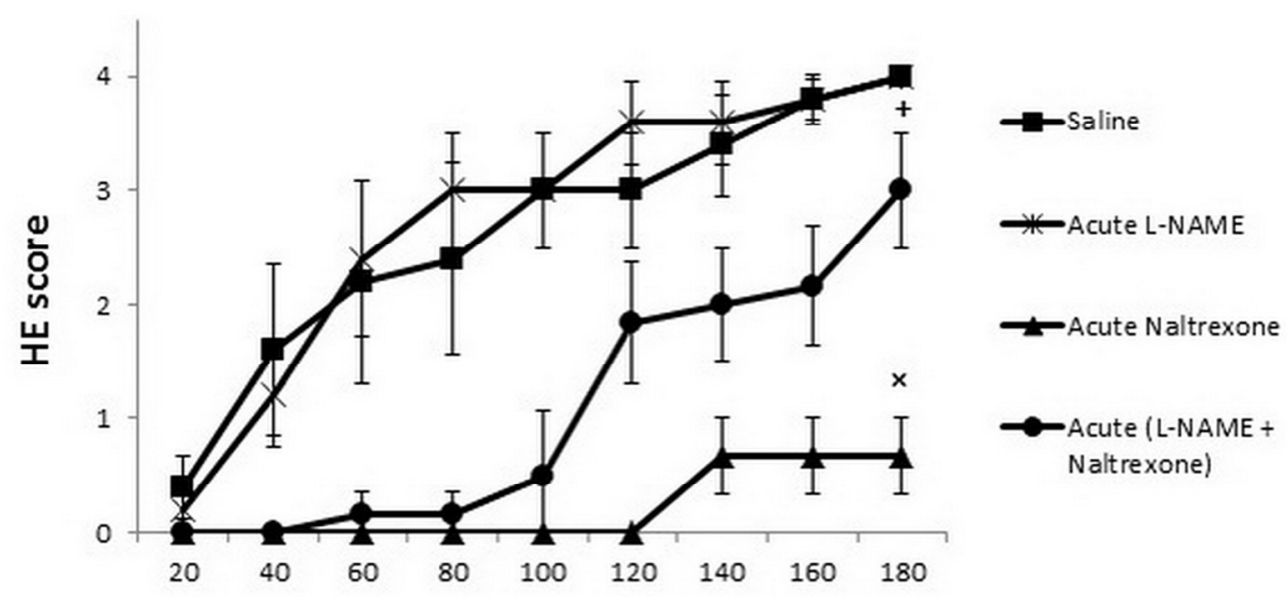

Time (minutes) after LPS injection

Fig. 2 Effects of chronic administration of naltrexone, L-NAME and co-administration of naltrexone and LNAME on LPS-induced hepatic encephalopathy in cirrhotic rats. Saline, Naltrexone (10 mg/kg, ip), and LNAME ( $3 \mathrm{mg} / \mathrm{kg}$, ip) were administered daily for 28 days after BDL. L-NAME ( $3 \mathrm{mg} / \mathrm{kg}), 15$ minutes before naltrexone $(10 \mathrm{mg} / \mathrm{kg})$ were administered daily for 28 days after surgery. One hour after the last injections on day 28 , LPS ( $1 \mathrm{mg} / \mathrm{kg}$, ip) was injected then hepatic encephalopathy grading was assessed throughout a

3-hour session. Data are presented as mean \pm S.E.M. Each group consisted of 8 rats and differences in hepatic encephalopathy scores were analyzed by repeated measures analysis of variance (ANOVA). $\times$ Significant differences between naltrexone- and saline-treated groups, 40-100 minutes after LPS injection (P $<0.01$ ) and 100-180 minutes after LPS injection $(P<0.001)$. + Significant differences between (L-NAME + naltrexone)- and saline-treated groups 40-100 minutes after LPS injection $(P<0.01)$ and $100-180$ minutes after LPS injection $(P<0.001)$.

$122 \times 67 \mathrm{~mm}(300 \times 300$ DPI $)$ 


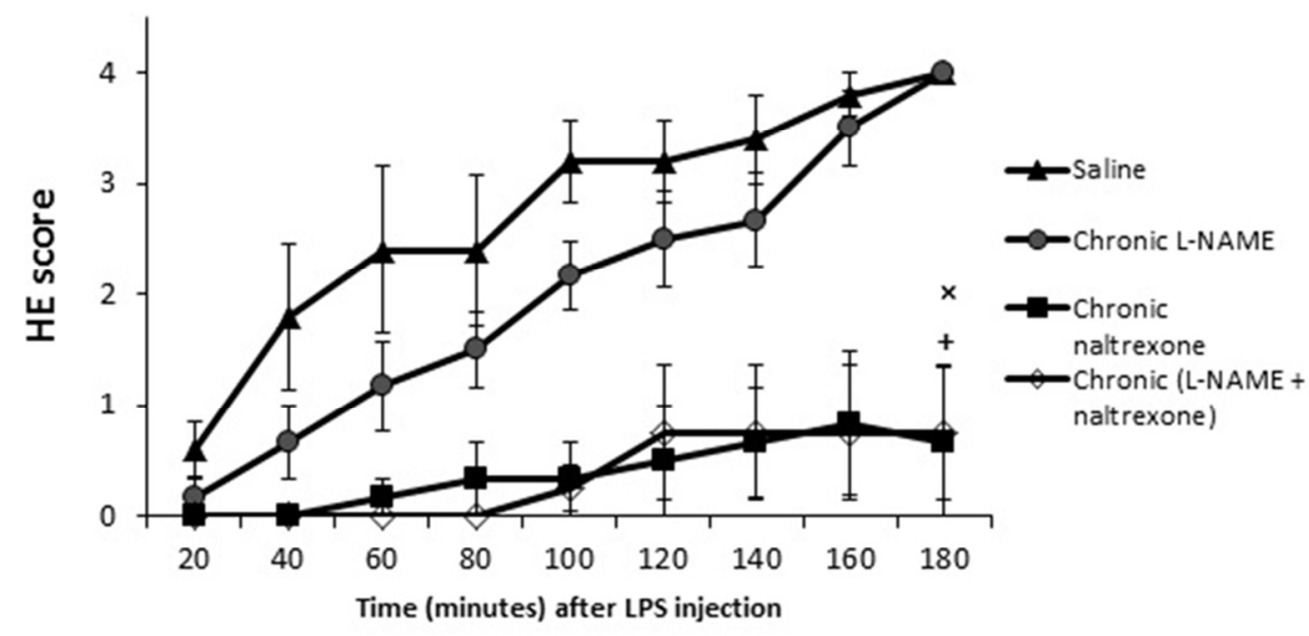

Fig. 3 Effects of acute administration of naltrexone, L-NAME and co-administration of naltrexone and L-NAME on LPS-induced hepatic encephalopathy in cirrhotic rats. Saline, Naltrexone (10 mg/kg, ip), and L-NAME (3 $\mathrm{mg} / \mathrm{kg}$, ip) were administered day 28 days. L-NAME (3 mg/kg), 15 minutes before naltrexone (10 mg/kg) were administered on day 28 after surgery. One hour after the injections, LPS ( $1 \mathrm{mg} / \mathrm{kg}$, ip) was injected then hepatic encephalopathy grading was assessed throughout a 3-hour session. Data are presented as mean \pm S.E.M. Each group consisted of 8 rats and differences in hepatic encephalopathy scores were analyzed by repeated measures analysis of variance (ANOVA). $\times$ Significant differences were observed between naltrexone- and saline-treated groups 40-100 minutes after LPS injection $(P<0.01)$ and $100-180$ minutes after LPS injection $(P<0.001)$. + Significant differences were observed between naltrexone- and saline-treated groups 100-180 minutes after LPS injection $(P<0.001)$.

$80 \times 43 \mathrm{~mm}(300 \times 300$ DPI $)$ 


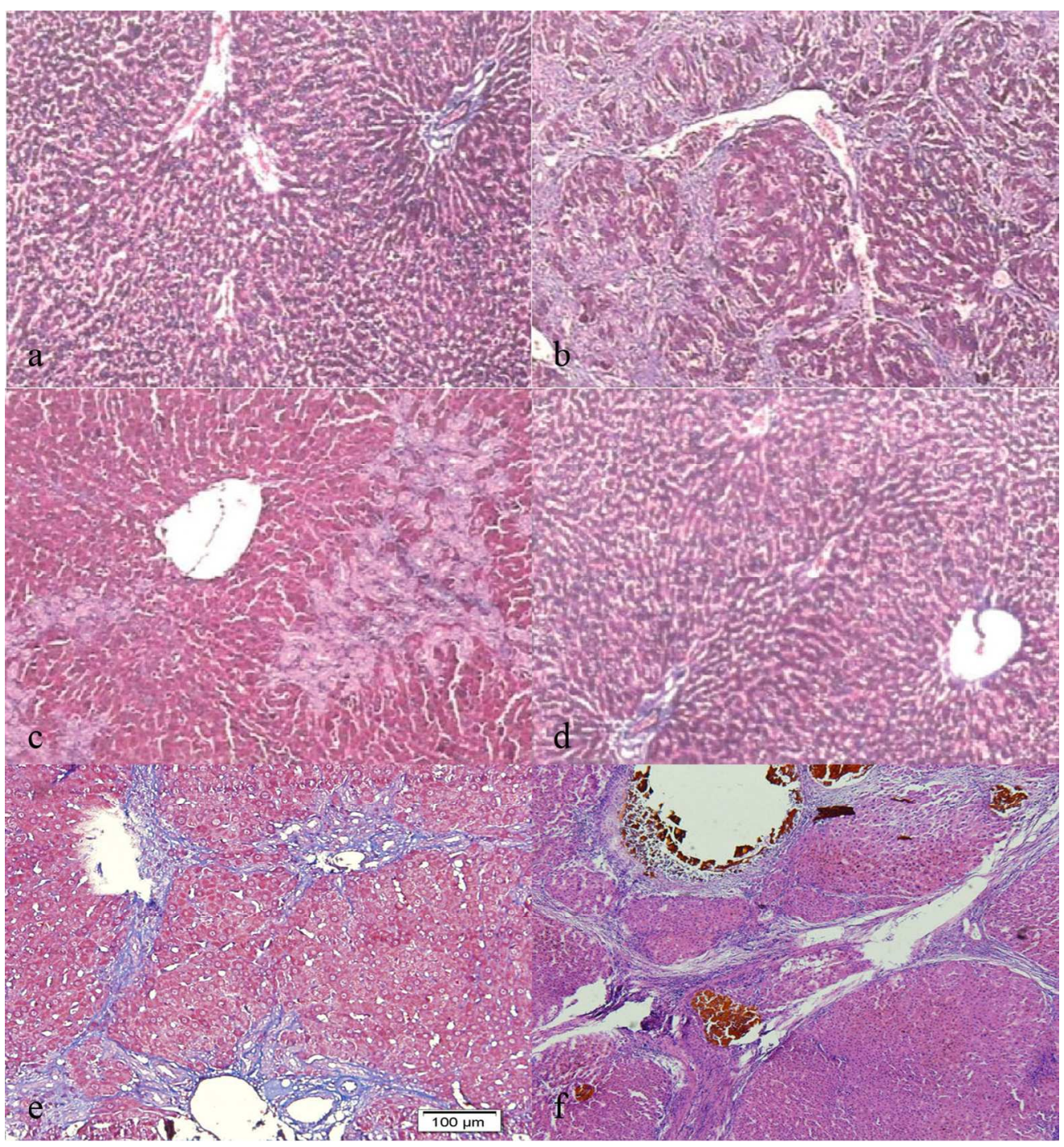

Fig. 4 Haematoxylin and eosin stained sections of sham and bile duct ligated (BDL) liver tissues in rats. Twenty eight days after the surgical procedure, liver tissue was obtained and collagen fibers were stained with Masson's Trichrome. Bile duct ligation was associated with extensive bridging fibrosis (portal to portal and portal to central linkage with fibrotic bands) and bile duct proliferation. A. Sham-operated rats, B. BDL rats, $C$. chronic treatment with naltrexone of BDL rats, D. acute treatment with naltrexone of BDL rats, E. acute treatment with L-NAME + naltrexone of BDL rats, and F. chronic treatment with L-NAME + naltrexone of BDL rats.

$493 \times 529 \mathrm{~mm}(300 \times 300 \mathrm{DPI})$ 